\title{
Oncogènes et récepteurs de facteurs de croissance
}

\author{
c-fms, v-fms et le récepteur de CSF-1 (ou M-CSF)
}

\section{Les nouvelles de ce numéro ont été préparées par : Axel Kahn Jean-Claude Dreyfus}

Il existe à l'heure actuelle trois récepteurs de facteurs de croissance bien caractérisés dont les gènes modifiés sont impliqués dans différents processus cancéreux : le récepteur à l'EGF (epidermal growth factor) auquel correspond le produit de l'oncogène v-erb $B$ qui en est une version tronquée, dépourvue du segment extracellulaire qui constitue le site de liaison d'EGF ; le récepteur des hormones thyroïdiennes $\left(m / s n^{\circ} 3\right.$, vol. $2, p .151$ et $n^{\circ} 3$, vol. 3, p. 172), codé par c-erb $\mathrm{A}$; le récepteur du facteur de croissance CSF-1 (ou M-CSF : monocytemacrophage colony stimulating factor), codé par c-fms. Le facteur M-CSF stimule la différenciation et la prolifération des cellules de la lignée monocytaire et fait partie des CSF, dont les autres membres sont GCSF (spécifique des granulocytes), GM-CSF (actif à la fois sur les granuleux et les monocytes), et multilineage CSF ou interleukine 3 (actif sur les différentes lignées hématopoiétiques... entre autres effets). Le récepteur de M-CSF est une glycoprotéine de membrane qui appartient à la famille des tyrosine-kinases ; la fixation du facteur de croissance au récepteur stimule l'autophosphorylation d'une tyrosine localisée du côté C-terminal de la protéine, c'est-à-dire dans sa portion cytoplasmique (tyrosine 969) [1]. Il est probable que la phosphorylation de cette tyrosine "inactive " le récepteur, constituant ainsi un efficace rétrocontrôle du stimulus prolifératif. Un rétrovirus provoquant des sarcomes chez le chat possède l'oncogène v-fms qui, lui aussi, code pour une glycoprotéine transmembranaire qui peut lier M-CSF, mais qui differe de c-fms à plusieurs niveaux. Les 40 derniers acides aminés $\mathrm{C}$-terminaux de c-fms sont notamment remplacés par 11 résidus totalement différents, ne comportant pas d'équivalent de la tyrosine 969. D'autres différences sont réparties dans la molécule. L'oncogène $v$-fms est transformant pour des fibroblastes alors que, même lorsque son expression est élevée, c-fms ne l'est pas. En revanche, la cotransfection des gènes codant pour c-fms et pour M-CSF conduit, bien qu'avec une faible efficacité, à la transformation cellulaire, preuve que la transduction du signal prolifératif est bien la cause de cette transformation. Les fibroblastes ne produisant pas de M-CSF, il apparaît de plus que, quoique liant le facteur de croissance, $v$-fms est transformant même en son absence.

Le rôle de la tyrosine 969 est précisé en construisant différents oncogènes hybrides ou mutés ; v-fms voit son pouvoir transformant diminué lorsque son extrémité C-terminale est remplacée par celle de cfms, sauf si la tyrosine 969 a été remplacée par une phénylalanine. La même mutation tyr $\rightarrow$ phe du résidu 969 ne suffit cependant pas à rendre transformant le récepteur produit de $\mathrm{c}-\mathrm{fms}$, mais augmente considérablement l'efficacité de la transformation lors de la cotransfection par c-fms ainsi modifié et M-CSF.

Toutes ces expériences indiquent que l'acquisition par v-fms de son pouvoir transformant est due, au moins, à deux types de modifications : des altérations, de localisation non précisée, rendant la transduction du signal indépendante de la fixation de M-CSF et l'absence de rétrocontrôle par perte du site phosphorylable à l'extrémité cytoplasmique de la molécule.
1. Sherr CJ. Growth factor receptors and cell
A.K.

$\mathrm{m} / \mathrm{s} n^{\circ} 8$ vol. 3 , octobre 87 
Lu cellules gliales, et plus spécifiquement les astrocytes, pourraient être infectables par le virus du SIDA HIV-I, telles sont les conclusions d'une récente étude portant sur l'infection in vitro de lignées en culture de cellules gliales. Les astrocytes sont considérés comme les cellules présentant l'antigène dans le cerveau, ce qui accroît les liens entre le virus et le système immunitaire. Il n'y a, cependant, pas de corrélation entre la sensibilité des lignées cellulaires à HIV et l'expression de la molécule CD4, suggérant qu'il existe d'autres structures cellulaires pouvant servir de récepteur au virus $(\mathrm{m} / \mathrm{s}$ $n^{\circ}$ 3, vol. 3, p. 180).

[Cheng-Mayer C, et al. Proc Natl Acad Sci USA 1987 ; 84 : 3526-30.]

Les principales données démographiques françaises des cinq dernières années sont résumées dans le tableau I ci-contre. Quelques remarques à formuler : l'indice de fécondité, bien que le plus élevé d'Europe occidentale, ne suffit pas à assurer le renouvellement des générations. La mortalité infantile continue à décroître. Le taux de nuptialité poursuit sa baisse, bien que de façon ralentie, les naissances hors mariage représentent en 1986 environ $20 \%$ du total. L'augmentation de la proportion des plus de 65 ans, inéluctable à terme, n'est pas apparente sur les cinq dernières années. Enfin, en ce qui concerne les étrangers, le solde migratoire, faible depuis 1983, est devenu nul en 1985 ; le pourcentage des enfants nés en France ayant au moins un parent étranger est voisin de 12 , le même qu'en 1976.

[Levy ML, Brouard N. Population et Sociétés, mars 1987, $\left.\mathrm{n}^{\circ} 211.\right]$

$\mathrm{m} / \mathrm{s} n^{\circ} 8$ vol. 3, octobre 87
口 L'acide rétinoïque pourrait être le morphogène dont le gradient contrôle la différenciation des digitations de l'aile du poulet lorsque du tissu de la marge postérieure du bourgeon embryonnaire de la future aile est greffé au niveau de la marge antérieure. Le produit pur mime cet effet et il existe, de fait, un gradient postero-antérieur d'acide rétinoïque dans le bourgeon. S'il s'avérait que l'acide rétinoïque est bien le morphogène "primaire ", ce serait la première molécule impliquée in vivo dans la morphogenèse d'un vertébré à être pleinement caractérisée.

[Thaller C, Eichele G. Nature 1987 ; 327 : 625-8.] la prise de nourriture !

[Flood JF, et al. Science 1987 ; 236 : 832-4.]

Manger augmente la mémoire ! Un repas pris immédiatement après un entraînement augmente significativement, chez la souris, la vitesse de mémorisation. L'effet du repas est mimé par l'injection intrapéritonéale de cholécystokinine...sauf si les nerfs vagues ont été sectionnés. Cet octapeptide se comporte donc comme un stimulateur périphérique des processus de mémorisation dont les effets centraux pourraient être dus à l'activation de fibres ascendantes des nerfs vagues. La signification évolutive de ce phénomène paraît claire : les animaux sauvages ont tout intérêt à mémoriser efficacement des comportements aboutissant à

Tableau I

FRANCE. INDICATEURS DÉMOGRAPHIQUES 1981-1986

\begin{tabular}{|c|c|c|c|c|c|c|}
\hline \multicolumn{7}{|c|}{$\begin{array}{l}\text { Tableau I } \\
\text { FRANCE. INDICATEURS DÉMOGRAPHIQUES 1981-1986 }\end{array}$} \\
\hline & 1981 & 1982 & 1983 & 1984 & 1985 & $\begin{array}{c}1986 \\
\text { Estimations }\end{array}$ \\
\hline $\begin{array}{l}\text { Naissances }(\mathrm{m}) \\
\text { Décès }(\mathrm{m}) \\
\text { Excédent naturel }(\mathrm{m}) \\
\text { Solde migratoire } \\
\text { Variation totale }(\mathrm{m})\end{array}$ & $\begin{array}{r}806 \\
555 \\
+251 \\
+\quad 56 \\
+\quad 307\end{array}$ & $\begin{array}{r}797 \\
543 \\
+\quad 254 \\
+\quad 37 \\
+\quad 291\end{array}$ & $\begin{array}{r}749 \\
560 \\
+\quad 189 \\
+\quad 16 \\
+\quad 205\end{array}$ & $\begin{array}{r}760 \\
542 \\
+\quad 217 \\
+\quad 14 \\
+\quad 234\end{array}$ & $\begin{array}{r}768 \\
552 \\
+216 \\
0 \\
+\quad 218\end{array}$ & $\begin{array}{r}778 \\
550 \\
+228 \\
+\quad 228\end{array}$ \\
\hline \multirow{2}{*}{$\begin{array}{l}\text { Taux de natalité }(t) \\
\text { Taux de mortalité }(t) \\
\text { Taux de mortalité } \\
\text { infantile }(t)\end{array}$} & $\begin{array}{l}14,9 \\
10,2\end{array}$ & $\begin{array}{l}14,6 \\
10,0\end{array}$ & $\begin{array}{l}13,7 \\
10,2\end{array}$ & $\begin{array}{r}13,8 \\
9,9\end{array}$ & $\begin{array}{l}13,9 \\
10,0\end{array}$ & $\begin{array}{l}14,0 \\
10,0\end{array}$ \\
\hline & 9,7 & 9,5 & 9,1 & 8,3 & 8,3 & 8,1 \\
\hline Indice de fécondité & 1,94 & 1,91 & 1,79 & 1,81 & 1,82 & 1,84 \\
\hline $\begin{array}{l}\text { Mariages }(\mathrm{m}) \\
\text { Taux de nuptialité }(\mathrm{t})\end{array}$ & $\begin{array}{l}315 \\
5,8\end{array}$ & $\begin{array}{r}312 \\
5,7\end{array}$ & $\begin{array}{r}300 \\
5,5\end{array}$ & $\begin{array}{r}281 \\
5,1\end{array}$ & $\begin{array}{l}270 \\
4,9\end{array}$ & $\begin{array}{l}226 \\
4,8\end{array}$ \\
\hline $\begin{array}{l}\text { Population (I) (m) } \\
\text { Moins de } 20 \text { ans (I) \% } \\
65 \text { ans ou plus (I) \% }\end{array}$ & $\begin{array}{r}54335 \\
30,0 \\
13,5\end{array}$ & $\begin{array}{r}54626 \\
29,8 \\
13,2\end{array}$ & $\begin{array}{r}54831 \\
29,4 \\
12,9\end{array}$ & $\begin{array}{r}55063 \\
29,1 \\
12,8\end{array}$ & $\begin{array}{r}55278 \\
28,8 \\
13,1\end{array}$ & $\begin{array}{r}55506 \\
28,5 \\
13,3\end{array}$ \\
\hline
\end{tabular}

(m) : milliers ; $(t)$ : taux pour 1000 habitants ; $(r)$ : taux pour 1000 naissances; (II): en fin d'année.
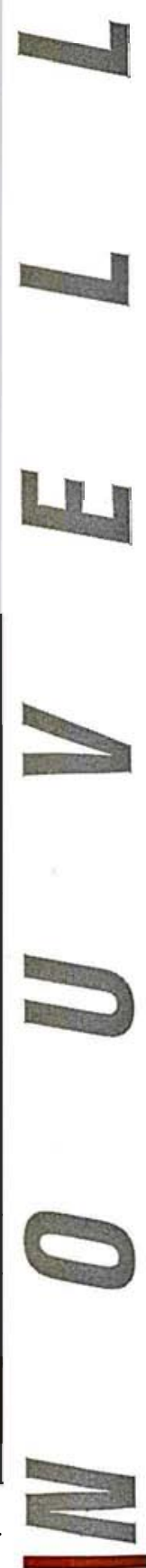